TRANSACTIONS OF THE

AMERICAN MATHEMATICAL SOCIETY

Volume 191, 1974

\title{
SPECTRAL ORDERS, UNIFORM INTEGRABILITY AND LEBESGUE'S DOMINATED CONVERGENCE THEOREM
}

\author{
BY
}

\section{KONG MING CHONG (1)}

ABSTRACT. Using the 'spectral' order relations < and $\ll$ introduced by Hardy, Littlewood and Pólya, we characterize the uniform integrability of a family of integrable functions. We also prove an extension and a 'converse' of the classical Lebesgue's dominated convergence theorem in terms of the 'spectral' orders $<$ and $\ll$.

1. Introduction. Denote by $M(X, \mu)$ the set of all extended real-valued measurable functions on a measure space $(X, \Lambda, \mu)$. Let $f \in M(X, \mu), g \in M\left(X^{\prime}, \mu^{\prime}\right)$, where $\mu(X)=\mu^{\prime}\left(X^{\prime}\right)$. Then $f$ and $g$ are said to be equimeasurable (written $f-g$ ) if $\mu\left(f^{-1}[I]\right)=\mu^{\prime}\left(g^{-1}[I]\right)$ for all bounded closed intervals $l$ of real numbers, where $I$ may be the singleton set $\{-\infty\}$ or $\{+\infty\}$.

If $f$ is any measurable (respectively nonnegative integrable) function defined on a finite (respectively infinite) measure space $(X, \Lambda, \mu)$, then there exists a unique right continuous decreasing function $\delta$, on the interval $[0, \mu(X)]$, called the decreasing rearrangement of $f$, such that $\delta_{f}$ and $f$ are equimeasurable. In fact

$$
\delta_{f}(t)=\inf \left\{s \in R: D_{f}(s) \leq t\right\}, \quad t \in[0, \mu(X)],
$$

where $D_{f}: R \rightarrow R$ is defined by $D_{f}(s)=\mu\{x \in X: f(x)>s\}, s \in R$.

In what follows, we shall denote the Lebesgue measure on $R$ by $m$.

If $f, g \in M(X, \mu) \cup M\left(X^{\prime}, \mu^{\prime}\right)$ and $f^{+}, g^{+} \in L^{1}(X, \mu) \cup L^{1}\left(X^{\prime}, \mu^{\prime}\right)$, where $\mu(X)$ $=\mu^{\prime}\left(X^{\prime}\right)=a<\infty$, then we write $f \ll g$ whenever

$$
\int_{0}^{t} \delta_{f} d m \leq \int_{0}^{t} \delta_{\boldsymbol{g}} d m, \quad t \in[0, a]
$$

and $f \prec g$ whenever $f \ll g$ and

Received by the editors April 4, 1973 and, in revised form, August 14, 1973.

AMS (MOS) subject classifications (1970). Primary 28A20.

Key words and phrases. Spectral orders, uniform integrability, equimeasurability, decreasing rearrangements, relatively weak compactness, nonatomic measure, closed convex hull, Lebesgue's dominated convergence theorem.

(1) This paper formed a part of the author's doctoral dissertation written under the guidance of Professor N. M. Rice and submitted to the Department of Mathematics, Queen's University in November, 1972. 


$$
\int_{0}^{a} \delta, d m=\int_{0}^{a} \delta_{\boldsymbol{g}} d m
$$

If $a=\mu(X)=\mu^{\prime}\left(X^{\prime}\right)$ is infinite, then the 'spectral' order relations $\prec$ and $\prec$ are defined for nonnegative integrable functions $f, g \in L^{1}(X, \mu) \cup L^{1}\left(X^{\prime}, \mu^{\prime}\right)$ analogously (we refer to $[1$, p. 53$]$ for an explanation of our adoption of the terminology 'spectral' order relations for $\ll$ and $\prec$ ).

Using a proof similar to that given in [5, p. 152], we can easily obtain the following.

Theorem 1.1 (Hardy, Littlewood and Pólya). (i) $f \ll g$ iff $\int(f-u)^{+} \leq \int(g-u)^{+}$ for all $u \in R$. (ii) $f<g$ iff $f \ll g$ and $\int f=\int g$.

2. Uniform integrability and decreasing rearrangements. Recall that a collection $\mathcal{C}$ of integrable functions defined on a finite measure space $(X, \Lambda, \mu)$ is said to be uniformly integrable if the limit

$$
\lim _{t \rightarrow \infty} \int_{\{|f|>t\}}|f| d \mu=0
$$

is uniform in $f \in \mathcal{C}$.

It is not hard to see that a family of integrable functions defined on a finite measure space is uniformly integrable if and only if the corresponding collection of their decreasing rearrangements is uniformly integrable. Thus uniform integrability is invariant under the operation of equimeasurable rearrangements.

The following theorem characterizes uniform integrability in terms of decreasing rearrangements.

Theorem 2.1. Suppose $(X, \Lambda, \mu)$ is a finite measure space with $\mu(X)=a$. Then a collection $\mathcal{C} \subset L^{1}(X, \mu)$ is uniformly integrable if and only if the limit

$$
\lim _{s \rightarrow 0} \int_{0}^{s} \delta|f| d m=0
$$

is uniform in $f \in \mathcal{C}$.

Moreover, if $C$ is uniformly integrable then $\delta_{|f|}(s)$ is uniformly bounded in $f \in \mathcal{C}$ for each $s$ satisfying $0<s \leq a$.

Proof. It is clear that $\mathcal{C}$ is uniformly integrable iff the collection $\left\{\delta_{|f|} \mid\right.$ $f \in \mathcal{C}_{\}}$is uniformly integrable since

$$
\int_{\{|f|>t\}}|f| d \mu=\int_{\{\delta|f|>t\}} \delta_{|f|} d m \text { for all } t \in R
$$

(cf. Corollary (1.9) in [1]).

Now for $t \geq 0$ and $s \in[0, \mu(X)]$, 


$$
\left.\left.\left.\int_{0}^{s}(\delta|f|-t) d m \leq \int(\delta|f|-t)^{+} d m=\int_{\{\delta|f|}>t\right\}{ }^{(\delta}|f|-t\right) d m \leq \int_{\{\delta|f|}>t\right\} \delta_{|f|} d m .
$$

Since $\left\{\delta_{|f|}: f \in \mathcal{C}\right\}$ is uniformly integrable, for any given $\epsilon>0$, there exists a $T \geq 0$ such that

$$
\left.\int_{\{\delta|f|}>T\right\} \delta_{|f|} d m<\epsilon / 2 \text { for all } f \in \mathcal{C} .
$$

Thus, for all $s$ satisfying $0 \leq s \leq \epsilon / 2 T$, we have

$$
\int_{0}^{s} \delta_{|f|} d m=\int_{0}^{s}[T+(\delta|f|-T)] d m<T s+\epsilon / 2 \leq \epsilon \text { for all } f \epsilon \mathcal{C} .
$$

Conversely, suppose that the condition holds. First we claim that $\sup \{\delta|f|(s)$ : $f \in \mathcal{C}\}$ is finite for each $s>0$. Suppose by contradiction that this is not the case. Then there is a point $s_{0}>0$ such that $\sup \left\{\delta_{|f|}\left(s_{0}\right): f \in \mathcal{C}\right\}=\infty$. Let $s_{n}=s_{0} / n$ where $n=1,2,3, \ldots$. Then, for each $n$, there exists an $f_{n} \in \mathcal{C}$ such that $\delta_{\left|f_{n}\right|}\left(s_{0}\right)>1 / s_{n}$ implying that

$$
\int_{0}^{s}{ }^{n} \delta_{\left|f_{n}\right|} d m \geq s_{n} \delta\left|f_{n}\right|_{n}^{\left(s_{n}\right) \geq s_{n} \delta\left|f_{n}\right|}\left(s_{0}\right)>1
$$

and so contradicting the uniform convergence of $\int_{0}^{s} \delta_{|f|} d m$ to 0 as $s \rightarrow 0$.

Next we claim that $\mu\{|f|>t\}$ can be made uniformly small as $t \rightarrow \infty$. To this end, we first note that the integrals $\int|f| d \mu$ for $f \in \mathcal{C}$ are uniformly bounded since

$$
\int|f| d \mu=\int_{0}^{a} \delta_{|f|} d m=\int_{0}^{s} \delta_{|f|} d m+\int_{s}^{a} \delta_{|f|} d m \leq \int_{0}^{s} \delta_{|f|} d m+(a-s) \delta_{|f|}(s)
$$

which is uniformly bounded in $f \in \mathcal{C}$ by virtue of the given condition and the preceding paragraph. Now $\mu\{|f|>t\} \leq t^{-1} \int_{\{|f|>t\}}|f| d \mu \leq t^{-1} \int|f| d \mu$ which can therefore be made uniformly small in $f \in \mathcal{C}_{\text {as }} t \rightarrow \infty$.

Finally, given any $\epsilon>0$, there exists an $s>0$ such that $\int_{0}^{s} \delta_{|f|} d m<\epsilon$ for all $f \in \mathcal{C}$. But there then exists a positive $T$ such that $\mu\{|f|>t\} \leq s$ for all $f \in \mathcal{C}$ and all $t>T$. Hence we have

$$
\int_{\{|f|>t\}}|f| d \mu=\int_{0}^{\mu\{|f|>t\}} \delta_{|f|} d m \leq \int_{0}^{s} \delta_{|f|} d m<\epsilon
$$

for all $f \in \mathcal{C}$ and for all $t>T$, showing that $\mathcal{C}$ is uniformly integrable. p. 18].

The following theorem gives a new proof of a well-known result given in [8,

Theorem 2.2. Let $\mathcal{H}$ and $\mathcal{K}$ be two uniformly integrable subsets of $L^{1}(X, \mu)$ where $\mu(X)^{\cdot}$ is finite. Then the sum $\mathcal{H}+\mathcal{K}$ is also uniformly integrable. 
Proof. By Theorem (2.1), we need only prove that

$$
\sup \left\{\int_{0}^{s} \delta_{|f+g|} d m: f \in \mathcal{H}, g \in \mathcal{K}\right\}
$$

converges to 0 as $s \rightarrow 0$. But, by Theorem 10.1 in [7, p. 108] (cf. [2, p. 88]) $\delta_{|f+g|} \leq \delta_{|f|+|g|}<\delta_{|f|}+\delta_{|8|}$ and so the result is immediate since both $\sup _{\{}\left\{\int_{0}^{s} \delta_{|f|} d m: f \in \mathcal{H}\right\}$ and $\sup _{2}\left\{\int_{0}^{s} \delta_{|g|} d m: g \in K\right\}$ converge to zero as $s \rightarrow 0$, by Theorem (2.1).

3. A natural setting for establishing uniform integrability. As a corollary to Theorem (2.1) we have the following theorem concerning the uniform integrability (and hence the relatively weak compactness) of a special collection of functions

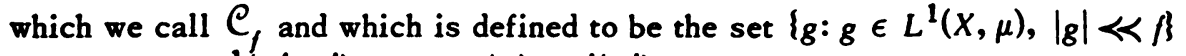
for some $f \in L^{1}\left(X^{\prime}, \mu^{\prime}\right)$ where $\mu(X)=\mu^{\prime}\left(X^{\prime}\right)<\infty$. As we shall see in the next section, the sets $\mathcal{C}_{f}$, where $f \in L^{1}\left(X^{\prime}, \mu^{\prime}\right)$ for some nonatomic finite measure $\mu^{\prime}$, characterize the uniformly integrable and hence relatively weakly compact subsets of $L^{1}(X, \mu)$ for all measure spaces $(X, \Lambda, \mu)$ with $\mu(X)=\mu^{\prime}\left(X^{\prime}\right)$; in fact, a subset of $L^{1}(X, \mu)$ is uniformly integrable if and only if it is a subset of $\mathcal{C}_{f}$ for some $f \in L^{1}\left(X^{\prime}, \mu^{\prime}\right)$. This theorem also contains as a particular case a theorem of $\mathrm{J} . \mathrm{V}$. Ryff [9, Theorem 2, p. 97] regarding the weak compactness of another special collection of functions which he called $\Omega_{f}$. Thus, the set $\mathcal{C}_{f}$, as well as the set $\boldsymbol{\Omega}_{\boldsymbol{f}}$, provides a natural setting in which to establish the uniform integrability of a family of functions.

Theorem 3.1. Suppose $(X, \Lambda, \mu)$ and $\left(X^{\prime}, \Lambda^{\prime}, \mu^{\prime}\right)$ are two measure spaces with equal finite total measures. If $0 \leq f \in L^{1}\left(X^{\prime}, \mu^{\prime}\right)$, then the set $\mathcal{C}_{f}=\left\{_{g}: g \in\right.$ $L^{1}(X, \mu),|g| \ll \beta$ is convex, uniformly integrable and $\sigma\left(L^{1}, L^{\infty}\right)$-closed.

Hence $C_{\text {, is a }} \sigma\left(L^{1}, L^{\infty}\right)$-compact convex set.

Proof. Assume that $f$ does not vanish almost everywhere, otherwise there is nothing to prove, for then $\mathcal{C}_{f}=\{0\}$ since $0 \leq g \prec \prec=0$ implies $g=0$ a.e.

The fact that $\mathcal{C}_{f}$ is convex is easy (cf. Theorem (4.3)(xv) in [1]).

To prove that $C_{f}$ is $\sigma\left(L^{1}, L^{\infty}\right)$-closed, we need only prove that it is $L^{1}$ closed since $\mathcal{C}_{f}$ is convex and $L^{1}$ is a convex space. It is also easy to see that $C_{\text {, is }} L^{1}$-closed (cf. Theorem (4.3)(xxiv) in [1]).

It remains to prove that $\mathcal{C}_{f}$ is uniformly integrable. This follows easily from Theorem (2.1) since $0 \leq \int_{0}^{s} \delta_{\mid g} \mid d m \leq \int_{0}^{s} \delta, d m$ for all $g \in \mathcal{C}_{f}$ and the last integral tends to 0 as $s$ tends to 0 by the absolute continuity of the integral of an integrable function, i.e. $\delta_{f}$.

The last assertion is an immediate consequence of the compactness criterion of Dunford and Pettis for uniform integrability [4, p. 294]. 
Corollary 3.2 (J. V. Ryff). Let $(X, \Lambda, \mu)$ and $\left(X^{\prime}, \Lambda^{\prime}, \mu^{\prime}\right)$ be finite measure spaces with $\mu(X)=\mu^{\prime}\left(X^{\prime}\right)$. If $f \in L^{1}\left(X^{\prime}, \mu^{\prime}\right)$, then the set $\Omega_{f}=\left\{g: g \in L^{1}(X, \mu)\right.$, $g<f\}$ is convex and $\sigma\left(L^{1}, L^{\infty}\right) \cdot$-compact.

Proof. We need only prove that $\Omega$, is a convex and $\sigma\left(L^{1}, L^{\infty}\right)$-closed subset of $\mathcal{C}_{|f|}$, since $\mathcal{C}_{|f|}$ is $\sigma\left(L^{1}, L^{\infty}\right)$-compact.

The convexity of $\Omega$, and the $\sigma\left(L^{1}, L^{\infty}\right)$-closedness of $\Omega$, follow as in $[7$, Theorem 15.3].

Finally, $\Omega_{f}$ is in fact a subset of $\mathcal{C}_{|f|}$ since, by Luxemburg's theorem $[7$, Theorem 9.5, p. 107], we have

$$
\Omega_{f}=\left\{g \in L^{1}(X, \mu): g<f\right\} \subset\left\{g \in L^{1}(X, \mu):|g| \ll|f|\right\}=\mathcal{C}_{|f|}
$$

Remark. A generalization of Ryff's theorem (i.e. Corollary (3.2)) was obtained by Luxemburg in [17, Theorem 15.3] for functions belonging to a rearrangement invariant Banach function space normed by a saturated Fatou norm.

It is not hard to see that the set $\mathcal{C}_{f}$ is well defined for integrable functions defined on infinite measure spaces, and that, in general, $\mathcal{C}_{f}$ is $\sigma\left(L^{1}, L^{\infty}\right)$-compact and convex.

4. Uniform integrability of functions and spectral orders. At this point, in view of Theorem (3.1) and its corollary, one may wonder, given a family $\mathcal{H}$ of uniformly integrable functions defined on a finite measure space $(x, \Lambda, \mu)$, whether there exists a positive integrable function $f$ defined on some possibly nonatomic measure space $\left(X^{\prime}, \Lambda^{\prime}, \mu^{\prime}\right)$ with $\mu^{\prime}\left(X^{\prime}\right)=\mu(X)$ such that $f$ dominates in the weak spectral order sense the modulus of every function in $\mathcal{H}$, i.e., $|g| \ll<f$ for all $g$ $\epsilon \mathcal{H}$ or equivalently, $\mathcal{H} \subset C_{f}$; and, if all the functions in $\mathcal{H}$ have equal integrals, whether there exists a function $f \in L^{1}\left(X^{\prime}, \mu^{\prime}\right)$ which dominates in the strong spectral order sense every function in $\mathcal{H}$, i.e. $g<f$ for all $g \in \mathcal{H}$ or equivalently, $\mathcal{H} \subset \Omega_{f}$. The answers to the above two questions are affirmative, as we shall see later in Theorem (4.2).

Lemma 4.1. Suppose $(X, \Lambda, \mu)$ is a given measure space, and $\left(X^{\prime}, \Lambda^{\prime}, \mu^{\prime}\right)$ is any nonatomic $\sigma$-finite measure space with the same (finite or infinite) total measure as $(X, \Lambda, \mu)$. Let $\mathcal{H} \subset L^{1}(X, \mu)$ be any family of functions. Then $\mathcal{H}$ satisfies

$$
\sup \left\{\int_{X}|g| d \mu: g \in \mathcal{H}\right\}<\infty
$$

and

$$
\sup _{X}\left\{\int_{X}(|g|-u)^{+} d \mu: g \in \mathcal{H}\right\} \rightarrow 0 \text { as } u \rightarrow \infty
$$


if and only if there exists a positive function $f \in L^{1}\left(X^{\prime}, \mu^{\prime}\right)$ sucb that $|g| \prec \prec f$ for all $g \in \mathcal{H}$, i.e. $H \subset \mathcal{C}_{f}$.

If, in addition, the measure spaces bave equal finite total measures and if the functions in $\mathcal{H}$ bave equal total integrals, the $\mathcal{H}$ satisfies the above conditions if and only if there exists a function $f \in L^{1}\left(X^{\prime}, \mu^{\prime}\right)$ sucb that $g<f$ for all $g \in \mathcal{H}$, i.e., $\mathcal{H} \subset \Omega_{f}$.

Proof. First, suppose $\mathcal{H}$ satisfies the conditions. Let $\mu(X)=\mu^{\prime}\left(X^{\prime}\right)=a$ where $a$ may be $\infty$. Let $G(s)=\sup \left\{\int_{0}^{s} \delta_{|g|} d m: g \in \mathcal{H}\right\}$ where $s \in[0, a]$ and let $\epsilon_{u}=\sup \left\{\int_{X}(|g|-u)^{+} d \mu: g \in \mathcal{H}\right\}$ for each $u>0$. Since $\int_{0}^{s} \delta_{|g|} d m \leq u s+\int_{X}(|g|-u)^{+} d u$ for all $s \in[0, a]$ and for all $u \geq 0$, we have

$$
G(s)=\sup _{\boldsymbol{g} \in \mathcal{H}} \int_{0}^{s} \delta_{\mid \boldsymbol{g}} \mid d m \leq u s+\sup _{\boldsymbol{g} \in \mathcal{H}} \int_{X}(|g|-u)^{+} d \mu .
$$

Thus if $L_{u}(s)=u s+\epsilon_{u}$, then $G(s) \leq L_{u}(s)$ where $s \in[0, a]$.

Define a function $F:[0, a] \rightarrow R^{+}$by $F(s)=\inf \left\{L_{u}(s): u \geq 0\right\}$ for $s \in[0, a]$. Then $F$ is well defined, since $L_{u}(s)$ is bounded by zero from below for each $s \epsilon$ $[0, a]$ and for each $u \geq 0$. Clearly, the function $F$ satisfies $G(s) \leq F(s)$ for all $s \in[0, a]$.

By definition, $F(s) \leq \sup _{g} \in \mathcal{H} \cdot \int_{X}|g| d \mu<\infty$ for all $s \in[0, a]$. We also have $F(0)=0$ since $\epsilon_{u} \rightarrow 0$ as $u$ tends to $\infty$ by hypothesis. Moreover, since each $L_{u}:[0, a] \rightarrow R^{+}$is a linear (and hence concave) increasing function for each fixed $u \geq 0$, it is not hard to see that the lower envelope $\inf \left\{L_{u}: u \geq 0\right\}$ of the collection $\left\{L_{u}: u \geq 0\right\}$ is concave and increasing, i.e., $F$ is a concave and increasing function on $[0, a]$. The derivative $F^{\prime}$ of $F$, therefore, exists almost everywhere on $[0, a]$ and is decreasing and positive. Without loss of generality, we may define $F^{\prime}$ to be right continuous. Furthermore, $F^{\prime}$ is integrable and $F(s)$ $=\int_{0}^{s} F^{\prime} d m$ where $s \in[0, a]$ since $F$ is concave and $0 \leq F \leq \sup _{g_{G} \in \mathcal{H}} \int|g| d \mu$ which is finite.

Since $G(s)=\sup _{g} \in \mathcal{H} \int_{0}^{s} \delta_{\mid \boldsymbol{B}} \mid d m \leq F(s)$, we have $\int_{0}^{s} \delta_{\mid \boldsymbol{g}} \mid d m \leq \int_{0}^{s} F^{\prime} d m$ for all $g$ $\epsilon \mathcal{H}$ and for all $s \in[0, a]$.

Hence $|g| \ll F^{\prime}$ for all $g \in \mathcal{H}$, i.e. $H \subset C_{F^{\prime}}=\left\{g \in L^{1}(X, \mu):|g| \ll F^{\prime}\right\}$.

Since $\left(X^{\prime}, \Lambda^{\prime}, \mu^{\prime}\right)$ is nonatomic and $\sigma$-finite, there exists a measure preserving transformation $\sigma: X^{\prime} \rightarrow[0, a]$, by Lorentz-Ryff-Day theorem $([6$, p. 60$]$, $[9$, Lemma 2, p. 95] and [3, Theorem (6.16), p. 39]). Define $f=F^{\prime} \circ \sigma$. Then $f \sim F^{\prime}$ (in fact, $\delta_{f}=F^{\prime}$ ) and hence $C_{F^{\prime}}=C_{f}$.

The converse of the preceding assertion is a trivial consequence of Theorem (1.1).

For the last assertion of the lemma, let $\mu(X)=\mu^{\prime}\left(X^{\prime}\right)=a<\infty$ and let 
$\int_{X} g d \mu=\alpha$ for all $g \in \mathcal{H}$ and for some constant $\alpha \in R$. Suppose $\mathcal{H}$ satisfies $\sup \left\{\int|g| d \mu: g \in \mathcal{H}\right\}<\infty$ and $\sup \left\{\int_{X}(|g|-u)^{+} d \mu: g \in \mathcal{H}\right\} \rightarrow 0$ as $u$ tends to $\infty$.

Since $\left(g^{+}-u\right)^{+} \leq(|g|-u)^{+}$and $\left(g^{-}-u\right)^{+} \leq(|g|-u)^{+}$, we also have $\sup \left\{\int_{X}\left(g^{+}-u\right)^{+} d \mu: g \in \mathcal{H}\right\} \rightarrow 0$ as well as $\sup \left\{\int_{X}\left(g^{-}-u\right)^{+} d \mu: g \in \mathcal{H}\right\} \rightarrow 0$ as $u$ $\rightarrow \infty$.

As before, we let, for all $u \in R, s \in[0, a]$,

$$
G(s)=\sup _{\boldsymbol{g} \in \mathcal{H}} \int_{0}^{s} \delta_{\boldsymbol{g}} d m \leq u s+\sup _{\boldsymbol{g} \in \mathcal{H}} \int_{X}(g-u)^{+} d \mu .
$$

Now if $u \geq 0$, then $\int_{X}(g-u)^{+} d \mu=\int_{X}\left(g^{+}-u\right)^{+} d \mu$. If $u<0$, then

$$
\begin{aligned}
\int_{X}(g-u)^{+} d \mu & =\int_{X}\left[(g-u)^{+}+u\right] d \mu-u a=\int_{X}\left[g^{+}-g^{-} \wedge(-u)\right] d \mu-u a \\
& =\int_{X}\left[g^{+}-g^{-} \wedge(-u)\right] d \mu+\int_{X} g d \mu-u a=\int_{X}\left(g^{-}+u\right)^{+} d \mu+a-u a .
\end{aligned}
$$

Hence $(*)$ implies

$$
G(s) \leq L_{u}(s)= \begin{cases}u s+\sup _{\boldsymbol{g} \in \mathcal{H}} \int_{X}\left(g^{+}-u\right)^{+} d \mu & \text { if } u \geq 0 \\ u(s-a)+\alpha+\sup _{\boldsymbol{g} \in \mathcal{H}} \int_{X}\left(g^{-}+u\right)^{+} d \mu & \text { if } u<0 .\end{cases}
$$

If we now follow exactly the same arguments as before, we arrive at a continuous concave function $F:[0, a] \rightarrow R$ which satisfies $F(0)=0, F(a)=a$ and $G \leq F=\inf \left\{L_{u}: u \in R\right\}$, and whose derivative $F^{\prime}$ satisfies $g \prec F^{\prime}$ for all $g \in \mathcal{H}$ since $F(a)=\int_{0}^{a} F^{\prime} d m=a$.

Hence $\mathcal{H} C \Omega_{F^{\prime}}=\left\{g \in L^{1}(X, \mu): g \prec F^{\prime}\right\}$.

Finally, since $\left(X^{\prime}, \Lambda^{\prime}, \mu^{\prime}\right)$ is nonatomic, by Lorentz-Ryff-Day theorem again, there exists a function $f \in L^{1}\left(X^{\prime}, \mu^{\prime}\right)$ such that $/ \sim F^{\prime}$. Thus $H \subset \Omega_{F^{\prime}}=\Omega_{f}$.

The following theorem gives the promised characterizations of uniform integrability of functions defined on a finite measure space in terms of spectral orders.

Theorem 4.2. Suppose $(X, \Lambda, \mu)$ is a finite measure space with $\mu(X)=a<\infty$ and $\left(X^{\prime}, \Lambda^{\prime}, \mu^{\prime}\right)$ is any nonatomic measure space with the same total measure. Let $\mathcal{H}$ be a family of integrable functions defined on $(X, \Lambda, \mu)$. Then the following are equivalent:

(i) $\mathcal{H}$ is uniformly integrable.

(ii) $\sup \left\{\int g \mid d \mu: g \in \mathcal{H}\right\}<\infty$ and $\sup \left\{\int(|g|-u)^{+} d \mu: g \in \mathcal{H}\right\} \rightarrow 0$ as $u \rightarrow \infty$.

(iii) There exists a positive function $f \in L^{1}\left(X^{\prime}, \mu^{\prime}\right)$ such that $H\left(C_{f}=\right.$ $\left\{g \in L^{1}(X, \mu):|g| \ll f\right\}$. 
If, in addition, the total integrals of the functions in $\mathcal{H}$ are equal, then state. ment (iii) above may be replaced by the following: $g<f$.

(iii)' There exists a function $f \in L^{1}\left(X^{\prime}, \mu^{\prime}\right)$ sucb that $\mathcal{H} \subset \Omega_{f}=\left\{g \in L^{\prime}(X, \mu)\right.$ :

Proof. We need only prove that (i) implies (ii), for the equivalence of (ii) and (iii) is proven in Lemma (4.1), and the implication (iii) $\Rightarrow$ (i) is contained in Theorem (3.1).

To prove (i) $\Rightarrow$ (ii), we first note that

$$
\int_{X}|g| d \mu=\int_{0}^{a} \delta_{|g|} d m \leq \int_{0}^{s} \delta_{|g|} d m+(a-s) \delta_{|g|}(s)
$$

for each $s$ satisfying $0<s \leq a$. Thus it is immediate from Theorem (2.1) that $\sup \left\{\int_{X}|g| d \mu: g \in \mathcal{H}\right\}<\infty$. Next, we observe that

$$
0 \leq \int_{X}(|g|-u)^{+}=\int_{\left\{\left.\right|_{g} \mid>u\right\}}(|g|-u) \leq \int_{\left\{\left.\right|_{B} \mid>u\right\}}|g|
$$

for all $u \geq 0$. Hence $\sup \left\{\int_{X}(|g|-u)^{+} d \mu: g \in \mathcal{H}\right\} \rightarrow 0$ as $u$ tends to $\infty$.

Corollary 4.3. Using the same notation as in Theorem (4.2), if $\mathcal{H} \subset L^{1}(X, \mu)$ is uniformly integrable, then $\bigcup\left\{\mathcal{C}_{|g|}: g \in \mathcal{H}\right\}$ is also uniformly integrable where $\mathcal{C}_{|g|}=\left\{b: b \in L^{1}(X, \mu)\right.$ and $\left.|b| \prec \prec|g|\right\}$.

Proof. Since there exists a function $f \in L^{1}\left(X^{\prime}, \mu^{\prime}\right)$ such that $H \subset C_{f}=$ $\left\{b \in L^{1}(X, \mu):|b| \longleftrightarrow<f\right\}$, we have $\bigcup\left\{\mathcal{C}_{\left.\right|_{g} \mid}: g \in \mathcal{H}\right\} \subset \mathcal{C}_{f}$.

In the following theorem, we give a new proof of a well-known fact given in [8, Theorem T20, p. 18] by showing that it is actually a particular case of Theorem (4.2).

Theorem 4.4. Let $\mathcal{H}$ be a uniformly integrable subset of $L^{1}(X, \mu)$, where $\mu(X)$ is finite. Then the closed convex bull of $\mathcal{H}$ is also uniformly integrable.

Proof. Let $\left(X^{\prime}, \Lambda^{\prime}, \mu^{\prime}\right)$ be any nonatomic measure space with $\mu^{\prime}\left(X^{\prime}\right)=\mu(X)$. Then, by Theorem (4.2), there exists an $f \in L^{1}\left(X^{\prime}, \mu^{\prime}\right)$ such that $\mathcal{H} \subset \mathcal{C}_{f}=\left\{g: g \in L^{1}(X, \mu)\right.$ and $|g| \prec \prec$. But, by Theorem (3.1), $\mathcal{C}_{f}$ is $L^{1}$-closed, convex and uniformly integrable, the closed convex hull of $\mathcal{H}$ is therefore again contained in $\mathcal{C}$, and so is also uniformly integrable.

5. An application of spectral orders to Lebesgue's dominated convergence theorem. In this section, we give an important application of the Hardy-LittlewoodPólya spectral order relations $\prec$ and $\prec$ to an extension of the classical Lebesgue's dominated convergence theorem. We show that, for integrable functions defined on a finite measure space, the theorem remains valid with the domination in the usual partial order sense $(\leq)$ replaced by domination in the weak spectral 
order sense ( $\prec)$. We also show that a converse of the theorem normally not achievable using the ordinary partial order $\leq$ is feasible through the use of the weak spectral order $\prec$.

The following is an extension of Lebesgue's dominated convergence theorem in terms of the spectral orders $\prec$ and $\prec$.

Theorem 5.1. Let $(X, \Lambda, \mu)$ be a finite measure space and let $\left(X^{\prime}, \Lambda^{\prime}, \mu^{\prime}\right)$ be any other nonatomic finite measure space with same total measure. Let $\left\{f_{n}: n \in N\right\}$ $C L^{1}(X, \mu)$ be a sequence of functions converging to a function $f \in M(X, \mu)$. Then the following are equivalent:

(a) There exists a positive function $g \in L^{1}\left(X^{\prime}, \mu^{\prime}\right)$ sucb that $\left|f_{n}\right| \prec \prec g, n=$ $1,2,3, \ldots$, i.e., $\left\{f_{n}: n \in N\right\} \subset \mathcal{C}_{g}$.

(b) $f \in L^{1}(X, \mu)$ and $f_{n} \rightarrow f$ in $L^{1}$ as $n \rightarrow \infty$.

If, in addition, the functions $f_{n}, \dot{n} \in N$, bave equal total integrals, then the following are equivalent:

(c) There exists a function $g \in L^{1}\left(X^{\prime}, \mu^{\prime}\right)$ sucb that $f_{n} \prec g, n \in N$, i.e., $\left\{f_{n}: n \in N\right\} \subset \Omega_{g}$.

(d) $f \in L^{1}(X, \mu)$ and $f_{n} \rightarrow f$ in $L^{1}$ as $n \rightarrow \infty$.

Proof. This is a direct consequence of Theorem (4.2) and a well-known extension of Lebesgue's dominated convergence theorem given, for example, in [8, Theorem T21, p. 18].

The following theorem gives a partial extension of Theorem (5.1) (i.e. a generalization of the implication (b) $\Rightarrow(a)$ in Theorem (5.1) for functions defined on infinite measure spaces).

Theorem 5.2. Suppose $(X, \Lambda, \mu)$ is a measure space and $\left(X^{\prime}, \Lambda^{\prime}, \mu^{\prime}\right)$ is any nonatomic $\sigma$-finite measure space with the same total measure as $(X, \Lambda, \mu)$. If $f$ and $f_{n}, n \in N$, are functions in $L^{1}(X, \mu)$ sucb that $f_{n} \rightarrow f$ in $L^{1}$ as $n \rightarrow \infty$, then there exists a nonnegative function $g \in L^{1}\left(X^{\prime}, \mu^{\prime}\right)$ such that $|f| \prec<g$ and $\left|f_{n}\right| \ll g$ for all $n \in N$, i.e. $f \in \mathcal{C}_{g}$ and $f_{n} \in \mathcal{C}_{\boldsymbol{g}}, n \in N$.

Proof. Since $\| f_{n}|-| f|| \leq\left|f_{n}-f\right|$, it is clear that $\left\|f_{n}\right\|_{1} \rightarrow\|f\|_{1}$ as $n \rightarrow \infty$ and so $\sup _{n \in N}\left\|f_{n}\right\|_{1}<\infty$. Moreover, $\left(\left|f_{n}\right|-u\right)^{+}$also converges to $(|f|-u)^{+}$in $L^{1}$ as $n \rightarrow \infty$ for all $u \geq 0$, since we have the inequality

$$
\left|\left(\left|f_{n}\right|-u\right)^{+}-(|f|-u)^{+}\right| \leq|| f_{n}|-| f|| \text {. }
$$

Let $\epsilon>0$ be given. Since $f \in L^{1}$, there exists $u_{1}$ such that $u \geq u_{1}$ implies $\int(|f|-u)^{+} \dot{d} \mu \leq \epsilon / 2$. Now there exists an $n_{1}$ such that $n \geq n_{1}$ implies

$$
\int\left(\left|f_{n}\right|-u\right)^{+} d \mu \leq \int\left(|f|-u_{1}\right)^{+} d \mu+\epsilon / 2 \leq \epsilon
$$

and so $u \geq u_{1}$ and $n \geq n_{1}$ imply $\int\left(\left|f_{n}\right|-u\right)^{+} d \mu \leq \int\left(|f|-u_{1}\right)^{+} d \mu \leq \epsilon$. Clearly, 
there exists $u_{2}$ such that $u \geq u_{2}$ implies that $\int\left(\left|f_{n}\right|-u\right)^{+} d \mu \leq \epsilon$ for all $1 \leq n \leq n_{1}$. Thus $u \geq \max \left(u_{1}, u_{2}\right)$ implies $\sup _{n} \in N\left\{\int\left(\left|f_{n}\right|-u\right)^{+} d \mu\right\} \leq \epsilon$, i.e. $\sup \left\{\int\left(\left|f_{n}\right|-u\right)^{+} d \mu\right.$ : $n \in N\} \rightarrow 0$ as $u \rightarrow \infty$. By Theorem (4.2), we therefore conclude that there exists a nonnegative function $g \in L^{1}\left(X^{\prime}, \mu^{\prime}\right)$ such that $f_{n} \in \mathcal{C}_{g}=\left\{b \in L^{1}(X, \mu):|b| \ll g\right\}$.

Finally, we also have $|f| \ll g$, since, by Fatou's lemma, we have, for all $u \geq 0$,

$$
\int(|f|-u)^{+} d \mu \leq \liminf _{n \rightarrow \infty} \int\left(\left|f_{n}\right|-u\right)^{+} \leq \int(g-u)^{+} d \mu .
$$

Before concluding this section, we give an example showing that the converse of Theorem (5.2) is not necessarily true for integrable functions defined on an infinite measure space, i.e., the implication $(a) \Rightarrow(b)$ in Theorem (5.1) does not necessarily hold if $\mu(X)=\infty$.

A counterexample. Let $f_{n}: R^{+} \rightarrow R^{+}, n \in N$, be a sequence of functions defined by $f_{n}=\chi[n-1, n), n \in N$. Then $f_{n} \rightarrow 0$ everywhere on $R^{+}$and $f_{n}<f_{1}=$ $\chi_{[0,1]}$ for all $n \in N$. But it is clear that the sequence does not converge to 0 in $L^{1}$.

\section{REFERENCES}

1. K. M. Chong, Equimeasurable rearrangements of functions with applications to analysis, Thesis, Queen's University, Kingston, Ontario, Canada, 1972.

2. K. M. Chong and N. M. Rice, Equimeasurable rearrangements of functions, Queen's Papers in Pure and Appl. Math., no. 28, 1971.

3. P. W. Day, Rearrangements of measurable functions, Thesis, Califomia Institute of Technology, 1970.

4. N. Dunford and J. T. Schwartz, Linear operators. I: General theory, Pure and Appl. Math., vol. 7, Interscience, New York, 1958. MR 22 \#3302.

5. G. H. Hardy, J. E. Littlewood and G. Pólya, Some simple inequalities satisfied by convex functions, Mess. of Math. 58 (1929), 145-152.

6. G. G. Lorentz, Bernstein polynomials, Mathematical Expositions, no. 8, Univ. of Toronto Press, Toronto, 1953. MR 15, 217.

7. W. A. J. Luxemburg, Rearrangement invariant Banach function spaces, Queen's Papers in Pure and Appl. Math. 10 (1967), 83-144.

8. P. A. Meyer, Probability and potentials, Blaisdell, Waltham, Mass., 1966. MR 34 \#5119.

9. J. V. Ryff, Orbits of $L^{1}$ functions under doubly stochastic transformations, Trans. Amer. Math. Soc. 117 (1965), 92-100. MR 35 \#762.

DEPARTMENT OF MATHEMATICS, UNIVERSITY OF MALAYA, KUALA LUMPUR, MALAYSIA 